Network Working Group

Request for Comments: 3887

Category: Standards Track
T. Hansen

AT\&T Laboratories

September 2004

\title{
Message Tracking Query Protocol
}

Status of this Memo

This document specifies an Internet standards track protocol for the Internet community, and requests discussion and suggestions for improvements. Please refer to the current edition of the "Internet Official Protocol standards" (STD 1) for the standardization state and status of this protocol. Distribution of this memo is unlimited.

Copyright Notice

Copyright (C) The Internet Society (2004).

Abstract

Customers buying enterprise message systems often ask: Can I track the messages? Message tracking is the ability to find out the path that a particular message has taken through a messaging system and the current routing status of that message. This document describes the Message Tracking Query Protocol that is used in conjunction with extensions to the ESMTP protocol to provide a complete message tracking solution for the Internet.

1. Introduction

The Message Tracking Models and Requirements document

[RFC-MTRK-MODEL] discusses the models that message tracking solutions could follow, along with requirements for a message tracking solution that can be used with the Internet-wide message infrastructure. This memo and its companions, [RFC-MTRK-ESMTP] and [RFC-MTRK-TSN], describe a complete message tracking solution that satisfies those requirements. The memo [RFC-MTRK-ESMTP] defines an extension to the SMTP service that provides the information necessary to track messages. This memo defines a protocol that can be used to query the status of messages that have been transmitted on the Internet via SMTP. The memo [RFC-MTRK-TSN] describes the message/tracking-status [RFC-MIME] media type that is used to report tracking status information. Using the model document's terminology, this solution uses active enabling and active requests with both request and chaining referrals. 


\subsection{Terminology}

The key words "MUST", "MUST NOT", "REQUIRED", "SHALL", "SHALL NOT", "SHOULD", "SHOULD NOT", "RECOMMENDED", "MAY", and "OPTIONAL" in this document are to be interpreted as described in BCP 14, RFC 2119 [RFC-KEYWORDS ] .

All syntax descriptions use the ABNF specified by [RFC-ABNF] . Terminal nodes not defined elsewhere in this document are defined in $[\mathrm{RFC}-\mathrm{ABNF}],[\mathrm{RFC}-\mathrm{URI}],[\mathrm{RFC}-\mathrm{MTRK}-\mathrm{ESMTP}],[\mathrm{RFC}-\mathrm{SMTP}]$, or $[\mathrm{RFC}-\mathrm{SMTPEXT}]$.

\section{Basic Operation}

The Message Tracking Query Protocol (MTQP) is similar to many other line-oriented Internet protocols, such as [POP3] and [NNTP]. Initially, the server host starts the MTQP service by listening on TCP port 1038 .

When an MTQP client wishes to make use of the message tracking service, it establishes a TCP connection with the server host, as recorded from the initial message submission or as returned by a previous tracking request. To find the server host, the MTQP client first does an SRV lookup for the server host using DNS SRV records, with a service name of "mtqp" and a protocol name of "tcp", as in mtqp._tcp.smtp3.example.com. (See the "Usage rules" section in [RFC-SRV] for details.) If the SRV records do not exist, the MTQP client then does an address record lookup for the server host. When the connection is established, the MTQP server sends a greeting. The MTQP client and MTQP server then exchange commands and responses (respectively) until the connection is closed or aborted.

\subsection{Tracking Service DNS Considerations}

Because of the ways server host lookups are performed, many different tracking server host configurations are supported.

A mail system that uses a single mail server host and has the MTQP server host on the same server host will most likely have a single MX record pointing at the server host, and if not, will have an address record. Both mail and MTQP clients will access that host directly.

A mail system that uses a single mail server host, but wants tracking queries to be performed on a different machine, MUST have an SRV MTQP record pointing at that different machine. 
A mail system that uses multihomed mail servers has two choices for providing tracking services: either all mail servers must be running tracking servers that are able to retrieve information on all messages, or the tracking service must be performed on one (or more) machine(s) that are able to retrieve information on all messages. In the former case, no additional DNS records are needed beyond the MX records already in place for the mail system. In the latter case, SRV MTQP records are needed that point at the machine(s) that are running the tracking service. In both cases, note that the tracking service MUST be able to handle the queries for all messages accepted by that mail system.

\subsection{Commands}

Commands in MTQP consist of a case-insensitive keyword, possibly followed by one or more parameters. All commands are terminated by a CRLF pair. Keywords and parameters consist of printable ASCII characters. Keywords and parameters are separated by whitespace (one or more space or tab characters). A command line is limited to 998 characters before the CRLF.

\subsection{Responses}

Responses in MTQP consist of a status indicator that indicates success or failure. Successful commands may also be followed by additional lines of data. All response lines are terminated by a CRLF pair and are limited to 998 characters before the CRLF. There are several status indicators: "+OK" indicates success; "+OK+" indicates a success followed by additional lines of data, a multiline success response; "-TEMP" indicates a temporary failure; "-ERR" indicates a permanent failure; and "-BAD" indicates a protocol error (such as for unrecognized commands).

A status indicator MAY be followed by a series of machine-parsable, case-insensitive response information giving more data about the errors. These are separated from the status indicator and each other by a single slash character ("/", decimal code 47). Following that, there MAY be white space and a human-readable text message. The human-readable text message is not intended to be presented to the end user, but should be appropriate for putting in a log for use in debugging problems.

In a multi-line success response, each subsequent line is terminated by a CRLF pair and limited to 998 characters before the CRLF. When all lines of the response have been sent, a final line is sent consisting of a single period (".", decimal code 046) and a CRLF pair. If any line of the multi-line response begins with a period, the line is "dot-stuffed" by prepending the period with a second 
period. When examining a multi-line response, the client checks to see if the line begins with a period. If so, and octets other than CRLF follow, the first octet of the line (the period) is stripped away. If so, and if CRLF immediately follows the period, then the response from the MTQP server is ended and the line containing the ".CRLF" is not considered part of the multi-line response.

An MTQP server MUST respond to an unrecognized, unimplemented, or syntactically invalid command by responding with a negative -BAD status indicator. A server MUST respond to a command issued when the session is in an incorrect state by responding with a negative -ERR status indicator.

\subsection{Firewall Considerations}

A firewall mail gateway has two choices when receiving a tracking query for a host within its domain: it may return a response to the query that says the message has been passed on, but no further information is available; or it may perform a chaining operation itself, gathering information on the message from the mail hosts behind the firewall, and returning to the MTQP client the information for each behind-the-firewall hop, or possibly just the final hop information, possibly also disguising the names of any hosts behind the firewall. Which option is picked is an administrative decision and is not further mandated by this document.

If a server chooses to perform a chaining operation itself, it MUST provide a response within 2 minutes, and SHOULD return a "no further information is available" response if it cannot provide an answer at the end of that time limit.

\subsection{Optional Timers}

An MTQP server MAY have an inactivity autologout timer. Such a timer MUST be of at least 10 minutes in duration. The receipt of any command from the client during that interval should suffice to reset the autologout timer. An MTQP server MAY limit the number of commands, unrecognized commands, or total connection time, or MAY use other criteria, to prevent denial of service attacks.

An MTQP client MAY have an inactivity autologout timer while waiting for a response from the server. Since an MTQP server may be a firewall, and may be chaining information from other servers, such a timer MUST be at least 2 minutes in duration. 


\section{Initialization and Option Response}

Once the TCP connection has been opened by an MTQP client, the MTQP server issues an initial status response that indicates its readiness. If the status response is positive (+OK or +OK+), the client may proceed with other commands.

The initial status response MUST include the response information "/MTQP". Negative responses MUST include a reason code as response information. The following reason codes are defined here; unrecognized reason codes added in the future may be treated as equivalent to "unavailable".

"/" "unavailable"

"/" "admin"

The reason code "/admin" SHOULD be used when the service is unavailable for administrative reasons. The reason code "/unavailable" SHOULD be used when the service is unavailable for other reasons.

If the server has any options enabled, they are listed as the multiline response of the initial status response, one per line. An option specification consists of an identifier, optionally followed by option-specific parameters. An option specification may be continued onto additional lines by starting the continuation lines with white space. The option identifier is case insensitive. Option identifiers beginning with the characters "vnd." are reserved for vendor use. (See below.)

One option specification is defined here:

STARTTLS [1*WSP "required"]

This capability MUST be listed if the optional STARTTLS command is enabled on the MQTP server and one or more certificates have been properly installed.

It has one optional parameter: the word "required" (The parameters for STARTTLS are case-insensitive). If the server requires that TLS be used for some of the domains the server handles, the server MUST specify the "required" parameter. 


\subsection{Examples}

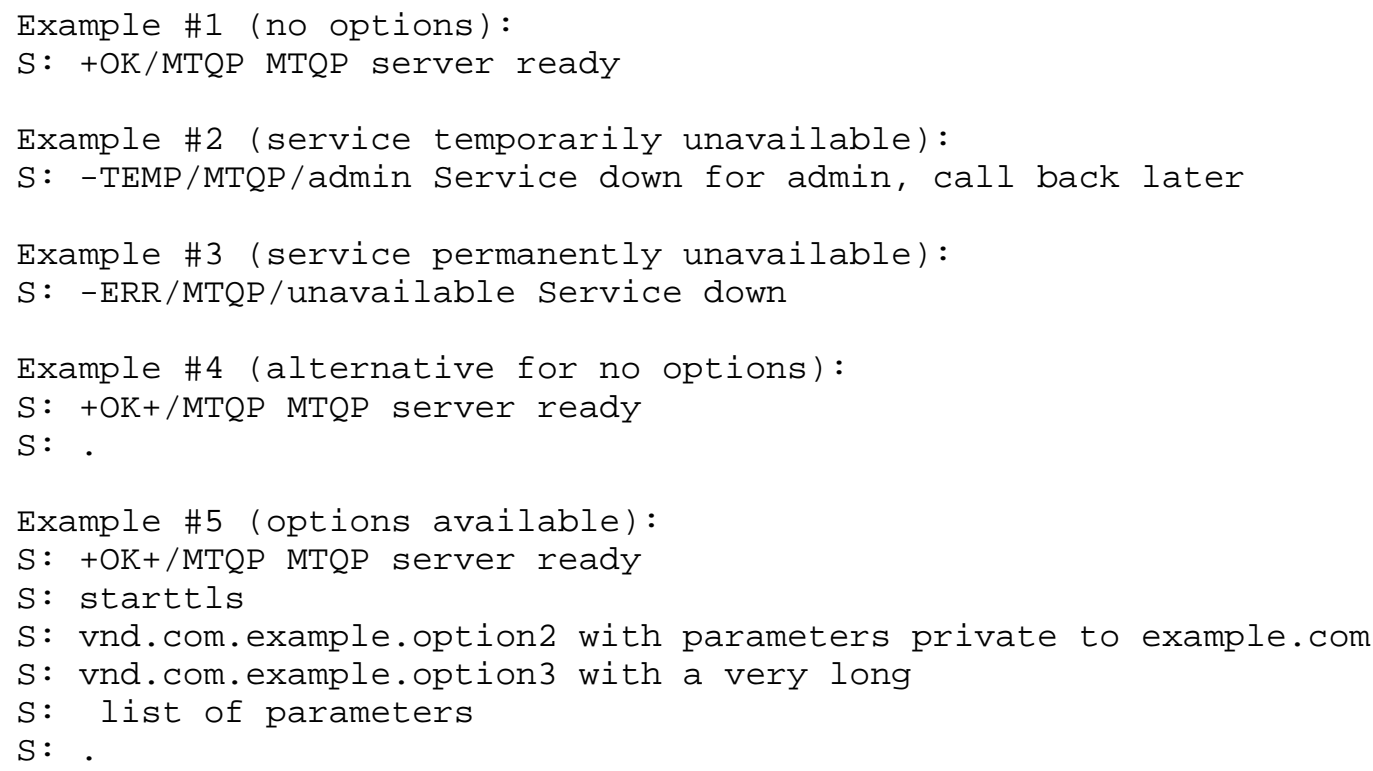

4. TRACK Command

Syntax :

track-command $=$ "TRACK" 1 *WSP unique-envid 1 *WSP mtrk-secret CRLF mtrk-secret $=$ base 64

Unique-envid is defined in [RFC-MTRK-ESMTP]. Mtrk-secret is the secret $A$ described in [RFC-MTRK-ESMTP], encoded using base64.

When the client issues the TRACK command, and the user is validated, the MTQP server retrieves tracking information about an email message. To validate the user, the value of mtrk-secret is hashed using SHA1, as described in [RFC-SHA1]. The hash value is then compared with the value passed with the message when it was originally sent. If the hash values match, the user is validated.

A successful response MUST be multi-line, consisting of a [RFC-MIME] body part. The MIME body part MUST be of type multipart/related, with subparts of message/tracking-status, as defined in [RFC-MTRK-TSN]. The response contains the tracking information about the email message that used the given tracking-id. A negative response to the TRACK command may include these reason codes: 


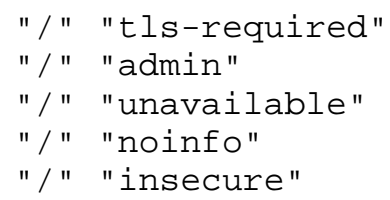

The reason code "/tls-required" SHOULD be used when the server has decided to require TLS. The reason code "/admin" SHOULD be used when the server has become unavailable, due to administrative reasons, since the connection was initialized. The reason code "/unavailable" SHOULD be used when the server has become unavailable, for other reasons, since the connection was initialized. The reason code "/insecure" is described later.

If a message has not been seen by the MTQP server, the server MUST choose between two choices: it MAY return a positive response with an action field of "opaque" in the tracking information, or it MAY return a negative response with a reason code of "noinfo".

\subsection{Examples}

In each of the examples below, the unique-envid is

"<12345-20010101dexample.com>", the secret A is "abcdefgh", and the SHA1 hash B is (in hex) "734ba8b31975d0dbae4d6e249f4e8da270796c94". The message came from example.com and the MTQP server is example2.com.

Example \#6 Message Delivered:

C: TRACK <12345-20010101@example.com> YWJjZGVmZ2gK

S: +OK+ Tracking information follows

S: Content-Type: multipart/related; boundary=으의 type=tracking-status

$S:$

$S:--\frac{\circ}{0} \div \circ \%$

S: Content-Type: message/tracking-status

$S:$

S: Original-Envelope-Id: 12345-20010101@example.com

S: Reporting-MTA: dns; example2.com

S: Arrival-Date: Mon, 1 Jan 2001 15:15:15 -0500

$\mathrm{S}:$

S: Original-Recipient: rfc822; userldexample1.com

S: Final-Recipient: rfc822; userldexample1.com

S: Action: delivered

S: Status: 2.5.0

$S:$

$S:--\frac{\circ}{5} \div \circ \circ \circ--$

$S:$. 


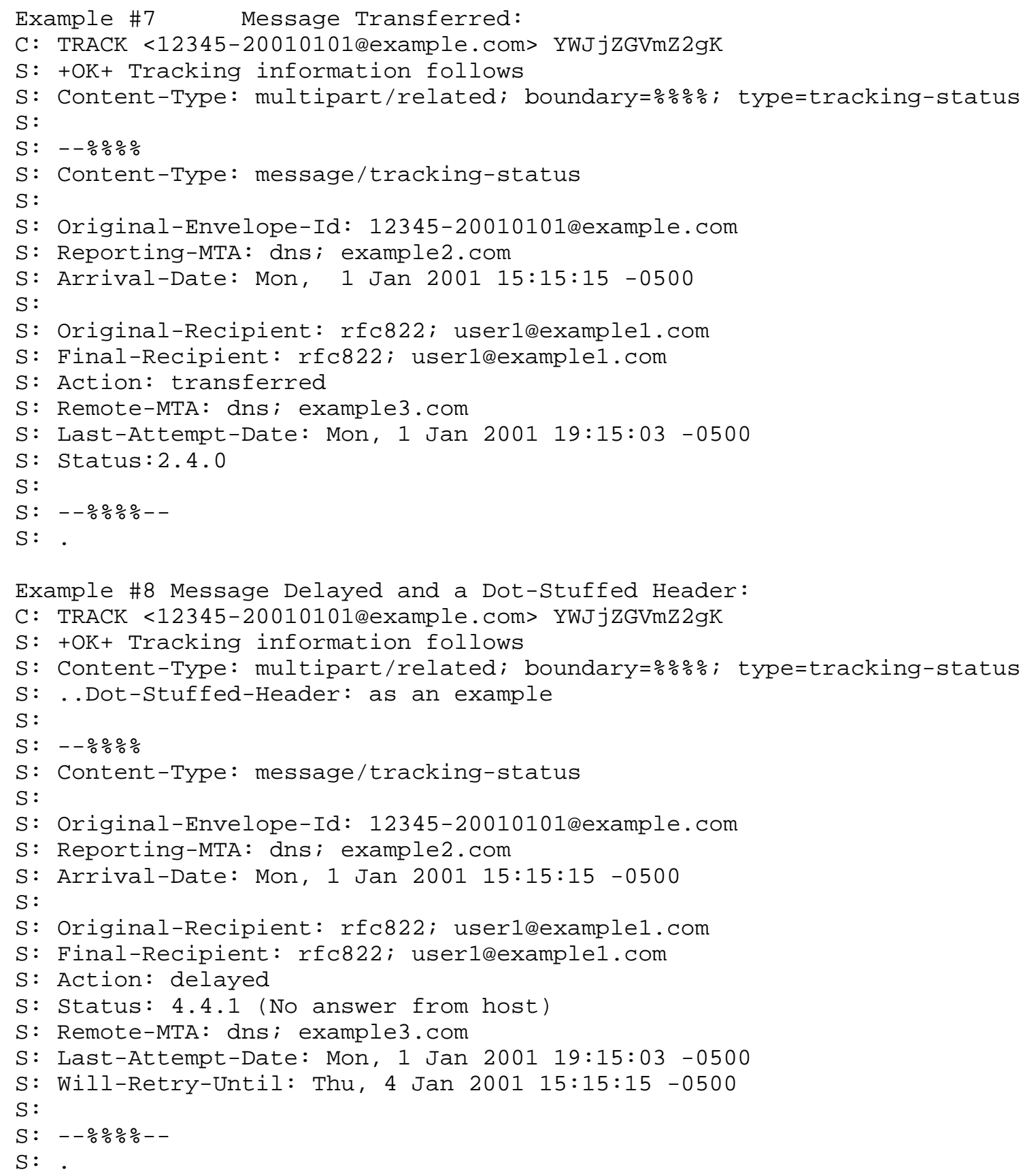




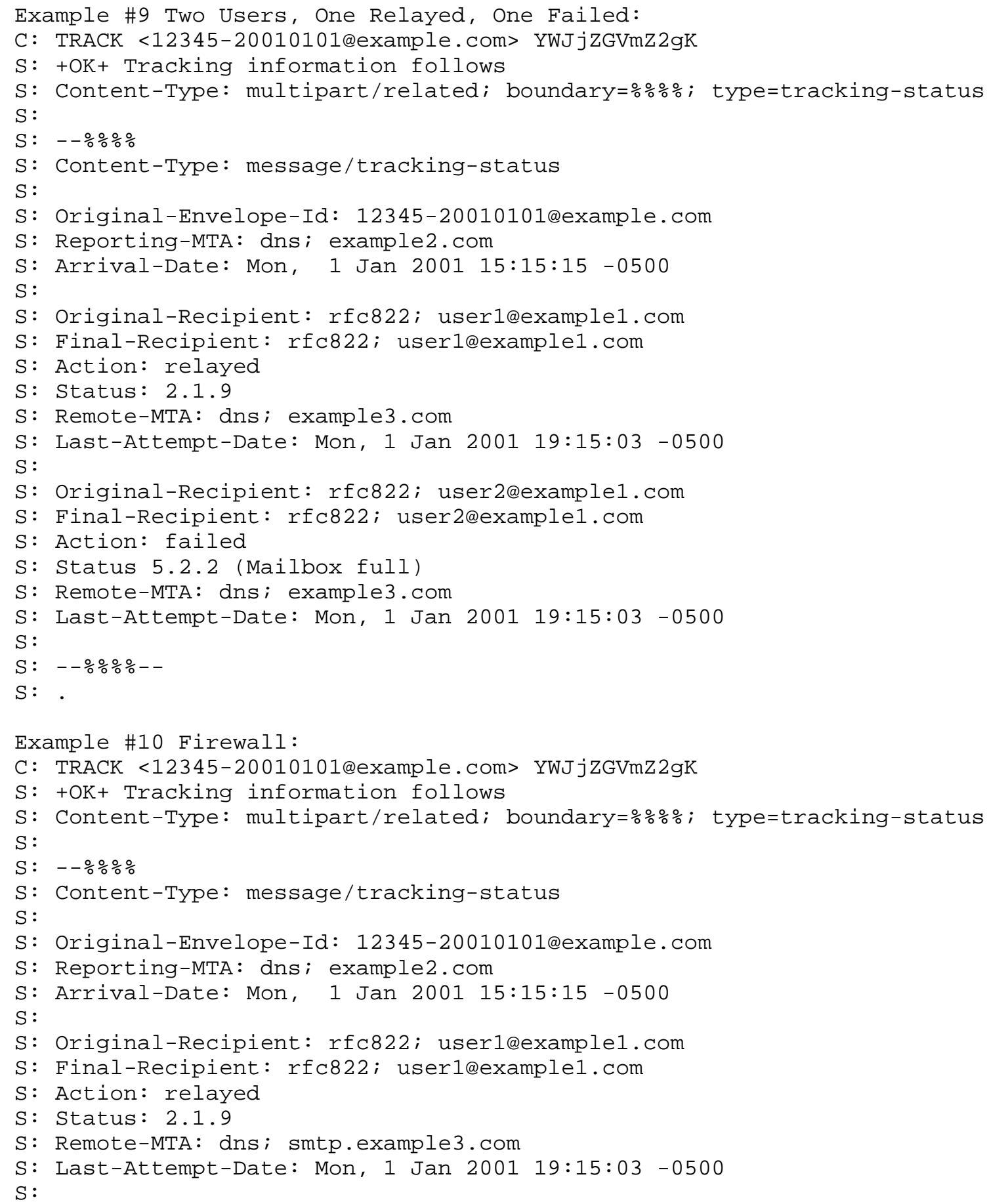




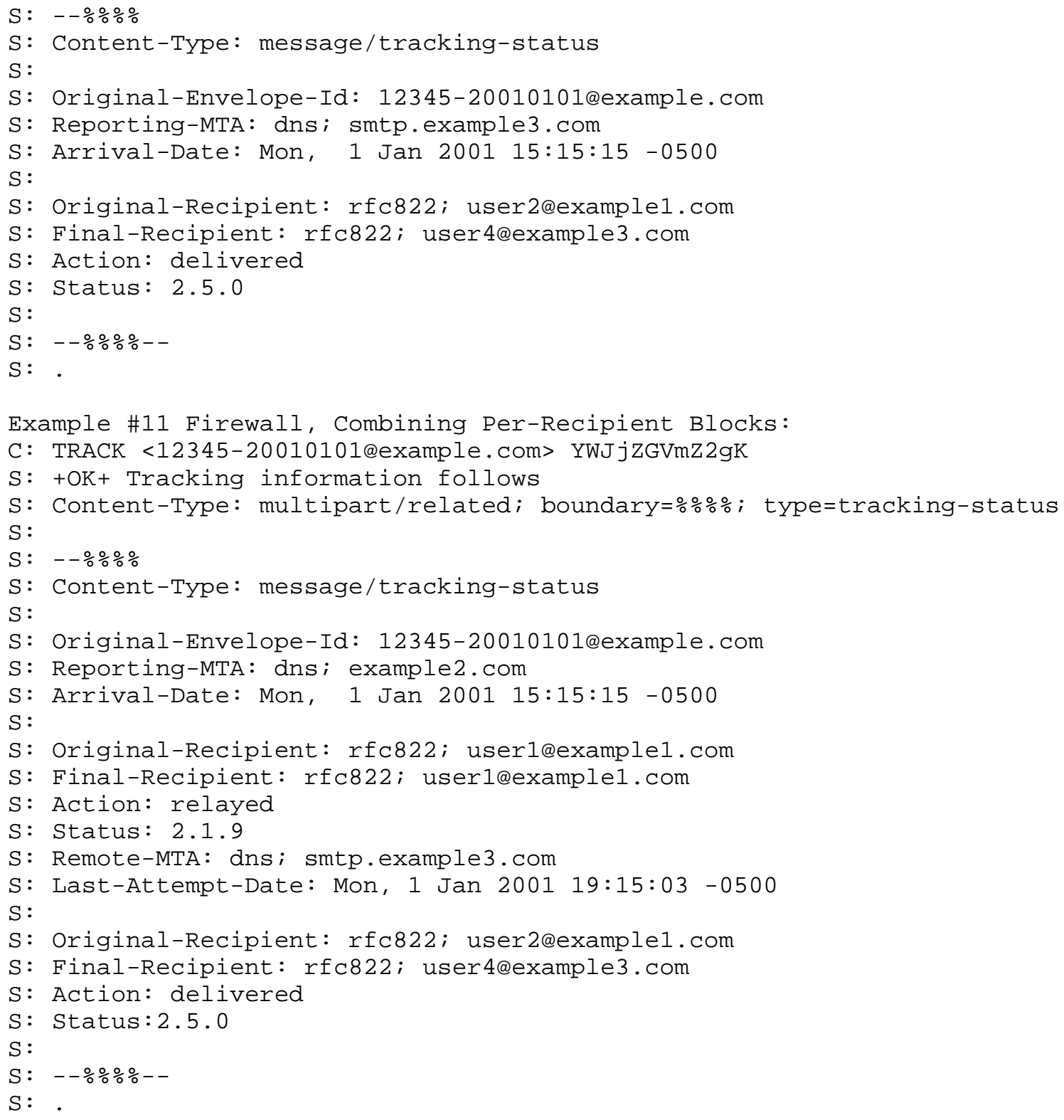




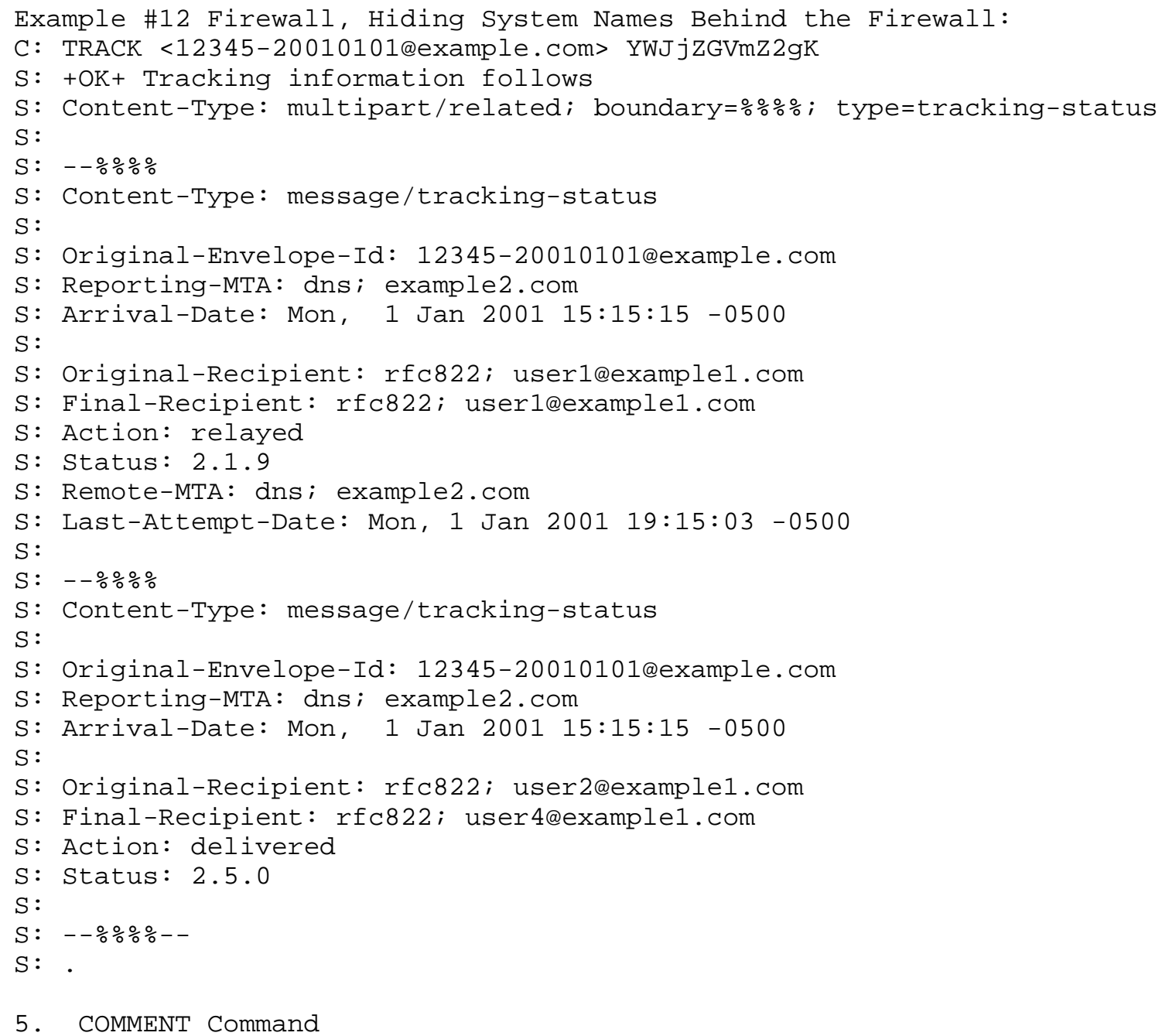

Syntax :

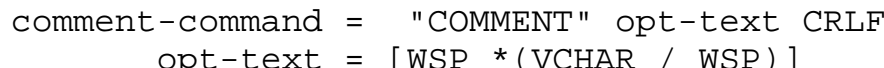

When the client issues the COMMENT command, the MTQP server MUST respond with a successful response (+OK or +OK+). All optional text provided with the COMMENT command are ignored. 


\section{STARTTLS Command}

Syntax :

starttls-command $=$ "STARTTLS" 1 *WSP domain *WSP CRLF domain $=($ sub-domain $1 *(" . "$ sub-domain $))$

TLS [TLS] is a popular mechanism for enhancing TCP communications with confidentiality and authentication. All MTQP servers MUST implement TLS. However, TLS MAY be disabled by a server administrator, either explicitly or by failing to install any certificates for TLS to use. If an MTQP server supports TLS and has one or more certificates available it MUST include "STARTTLS" in the option specifications list on protocol startup.

Note: TLS SHOULD be enabled on MQTP servers whenever possible.

The parameter MUST be a fully qualified domain name (FQDN). A client MUST specify the hostname it believes it is speaking with so that the server may respond with the proper TLS certificate. This is useful for virtual servers that provide message tracking for multiple domains (i.e., virtual hosting).

If the server returns a negative response, it MAY use one of the following response codes:

" /" "unsupported"

"/" "unavailable"

"/" "tls-in-progress"

" / "bad-fqdn"

If TLS is not supported, then a response code of "/unsupported" SHOULD be used. If TLS is not available for some other reason, then a response code of "/unavailable" SHOULD be used. If a TLS session is already in progress, then it is a protocol error and "-BAD" MUST be returned with a response code of "/tls-in-progress". If there is a mismatch between the supplied FQDN and the FQDN found in the dNSName field of the subjectAltName extension of the server's certificate [RFC-X509], then it is a protocol error and "-BAD" MUST be returned with a response code of "/bad-fqdn".

After receiving a positive response to a STARTTLS command, the client MUST start the TLS negotiation before giving any other MTQP commands.

If the MTQP client is using pipelining (see below), the STARTTLS command must be the last command in a group. 
6.1. Processing After the STARTTLS Command

If the TLS handshake fails, the server SHOULD abort the connection.

After the TLS handshake has been completed, both parties MUST immediately decide whether or not to continue based on the authentication and confidentiality achieved. The MTQP client and server may decide to move ahead even if the TLS negotiation ended with no authentication and/or no confidentiality because most MTQP services are performed with no authentication and no confidentiality, but some MTQP clients or servers may want to continue only if a particular level of authentication and/or confidentiality was achieved.

If the MTQP client decides that the level of authentication or confidentiality is not high enough for it to continue, it SHOULD issue an MTQP QUIT command immediately after the TLS negotiation is complete.

If the MTQP server decides that the level of authentication or confidentiality is not high enough for it to continue, it MAY abort the connection. If it decides that the level of authentication or confidentiality is not high enough for it to continue, and it does not abort the connection, it SHOULD reply to every MTQP command from the client (other than a QUIT command) with a negative "-ERR" response and a response code of "/insecure".

\subsection{Result of the STARTtLS Command}

Upon completion of the TLS handshake, the MTQP protocol is reset to the initial state (the state in MTQP after a server starts up). The server MUST discard any knowledge obtained from the client prior to the TLS negotiation itself. The client MUST discard any knowledge obtained from the server, such as the list of MTQP options, which was not obtained from the TLS negotiation itself.

At the end of the TLS handshake, the server acts as if the connection had been initiated and responds with an initial status response and, optionally, a list of server options. The list of MTQP server options received after the TLS handshake MUST be different than the list returned before the TLS handshake. In particular, a server MUST NOT return the STARTTLS option in the list of server options after a TLS handshake has been completed.

Both the client and the server MUST know if there is a TLS session active. A client MUST NOT attempt to start a TLS session if a TLS session is already active. 
7. QUIT Command

Syntax :

quit-command = "QUIT" CRLF

When the client issues the QUIT command, the MTQP session terminates. The QUIT command has no parameters. The server MUST respond with a successful response. The client MAY close the session from its end immediately after issuing this command (if the client is on an operating system where this does not cause problems).

8. Pipelining

The MTQP client may elect to transmit groups of MTQP commands in batches without waiting for a response to each individual command. The MTQP server MUST process the commands in the order received.

Specific commands may place further constraints on pipelining. For example, STARTTLS must be the last command in a batch of MTQP commands.

8.1. Examples

The following two examples are identical:

Example \#13:

C: TRACK <tracking-id> YWJjZGVmZ2gK

$S:$ +OK+ Tracking information follows

$S:$

S: ... tracking details \#1 go here ...

$S:$.

C: TRACK <tracking-id-2> QUJDREVGR0gK

S: +OK+ Tracking information follows

$S:$

S: ... tracking details \#2 go here ...

$\mathrm{S}:$. 


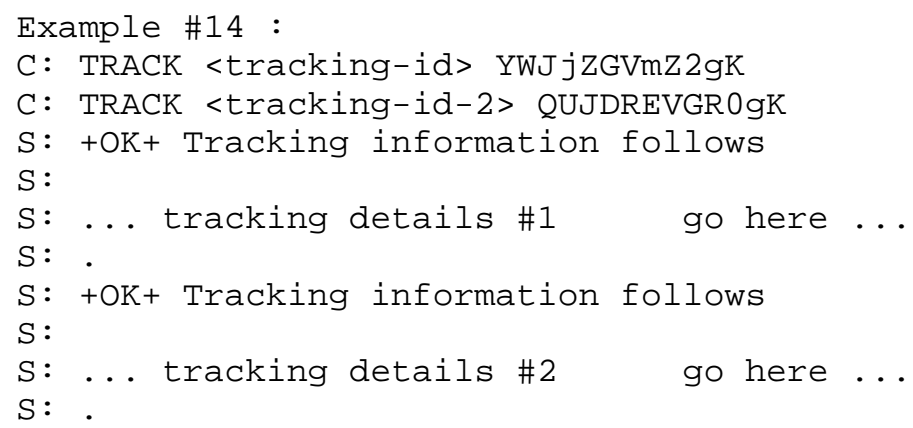

9. The MTQP URI Scheme

9.1. Intended usage

The MTQP URI scheme is used to designate MTQP servers on Internet hosts accessible using the MTQP protocol. It performs an MTQP query and returns tracking status information.

9.2. URI Scheme Name

The name of the URI scheme is "mtqp".

9.3. URI Scheme Syntax

An MTQP URI takes one of the following forms:

mtqp: / / <mserver $>/$ track / <unique-envid $>/<$ mtrk-secret $>$

mtqp: / / <mserver $>$ : <port>/track/<unique-envid > / mtrk-secret>

The first form is used to refer to an MTQP server on the standard port, while the second form specifies a non-standard port. Both of these forms specify that the TRACK command is to be issued using the given tracking id (unique-envid) and authorization secret (mtrksecret). The path element "/track/" MUST BE treated case insensitively, but the unique-envid and mtrk-secret MUST NOT be.

\subsubsection{Formal Syntax}

This is an ABNF description of the MTQP URI.

mtqp-uri = "mtqp://" authority "/track/" unique-envid "/" mtrk-secret 


\subsection{Encoding Rules}

The encoding of unique-envid is discussed in [RFC-MTRK-ESMTP] . Mtrk-secret is required to be base64 encoded. If the "/", "?" and "ㅇㅇ octets appear in unique-envid or mtrk-secret, they are further required to be represented by a "\%" followed by two hexadecimal characters. (The two characters give the hexadecimal representation of that octet).

10. IANA Considerations

System port number 1038 has been assigned to the Message Tracking Query Protocol by the Internet Assigned Numbers Authority (IANA) .

The service name "MTQP" has been registered with the IANA.

The IANA has also registered the URI registration template found in Appendix A in accordance with [BCP35].

This document requests that IANA maintain one new registry: MTQP options. The registry's purpose is to register options to this protocol. Options whose names do not begin with "vnd." MUST be defined in a standards track or IESG approved experimental RFC. New MTQP options MUST include the following information as part of their definition:

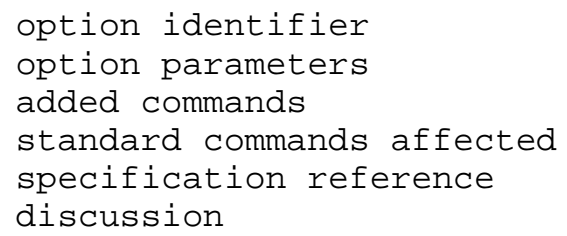

One MTQP option is defined in this document, with the following registration definition:

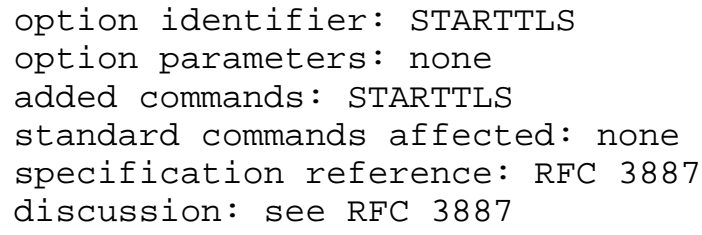

Additional vendor-specific options for this protocol have names that begin with "vnd.". After the "vnd." would appear the reversed domain name of the vendor, another dot ".", and a name for the option itself. For example, "vnd.com.example.extinfo" might represent a 
vendor-specific extension providing extended information by the owner of the "example.com" domain. These names MAY be registered with IANA.

\section{Security Considerations}

If the originator of a message were to delegate his or her tracking request to a third party, this would be vulnerable to snooping over unencrypted sessions. The user can decide on a message-by-message basis if this risk is acceptable.

The security of tracking information is dependent on the randomness of the secret chosen for each message and the level of exposure of that secret. If different secrets are used for each message, then the maximum exposure from tracking any message will be that single message for the time that the tracking information is kept on any MTQP server. If this level of exposure is too much, TLS may be used to reduce the exposure further.

It should be noted that message tracking is not an end-to-end mechanism. Thus, if an MTQP client/server pair decide to use TLS confidentiality, they are not securing tracking queries with any prior or successive MTQP servers.

Both the MTQP client and server must check the result of the TLS negotiation to see whether acceptable authentication or confidentiality was achieved. Ignoring this step completely invalidates using TLS for security. The decision about whether acceptable authentication or confidentiality was achieved is made locally, is implementation-dependent, and is beyond the scope of this document.

The MTQP client and server should note carefully the result of the TLS negotiation. If the negotiation results in no confidentiality, or if it results in confidentiality using algorithms or key lengths that are deemed not strong enough, or if the authentication is not good enough for either party, the client may choose to end the MTQP session with an immediate QUIT command, or the server may choose to not accept any more MTQP commands.

A man-in-the-middle attack can be launched by deleting the "STARTTLS" option response from the server. This would cause the client not to try to start a TLS session. An MTQP client can protect against this attack by recording the fact that a particular MTQP server offers TLS during one session and generating an alarm if it does not appear in an option response for a later session. 
Similarly, the identity of the server as expressed in the server's certificate should be cached, and an alarm generated if they do not match in a later session.

If TLS is not used, a tracking request is vulnerable to replay attacks, such that a snoop can later replay the same handshake again to potentially gain more information about a message's status.

Before the TLS handshake has begun, any protocol interactions are performed in the clear and may be modified by an active attacker. For this reason, clients and servers MUST discard any knowledge obtained prior to the start of the TLS handshake upon completion of the TLS handshake.

If a client/server pair successfully performs a TLS handshake and the server does chaining referrals, then the server sHould attempt to negotiate TLS at the same (or better) security level at the next hop. In a hop-by-hop scenario, STARTTLS is a request for "best effort" security and should be treated as such.

SASL is not used because authentication is per message rather than per user.

12. Protocol Syntax

This is a collected ABNF description of the MTQP protocol. mtqp-uri = "mtqp://" authority "/track/" unique-envid "/" mtrk-secret conversation $=$ command-response * (client-command command-response)

; client side

client-command = track-command / starttls-command / quit-command

/ comment-command

track-command = "TRACK" 1 *WSP unique-envid 1 *WSP mtrk-secret CRLF mtrk-secret $=$ base 64

starttls-command $=$ "STARTTLS" 1 *WSP domain *WSP CRLF

domain $=($ sub-domain $1 *(" . "$ sub-domain $))$

quit-command = "QUIT" CRLF

comment-command $=$ "COMMENT" opt-text CRLF 


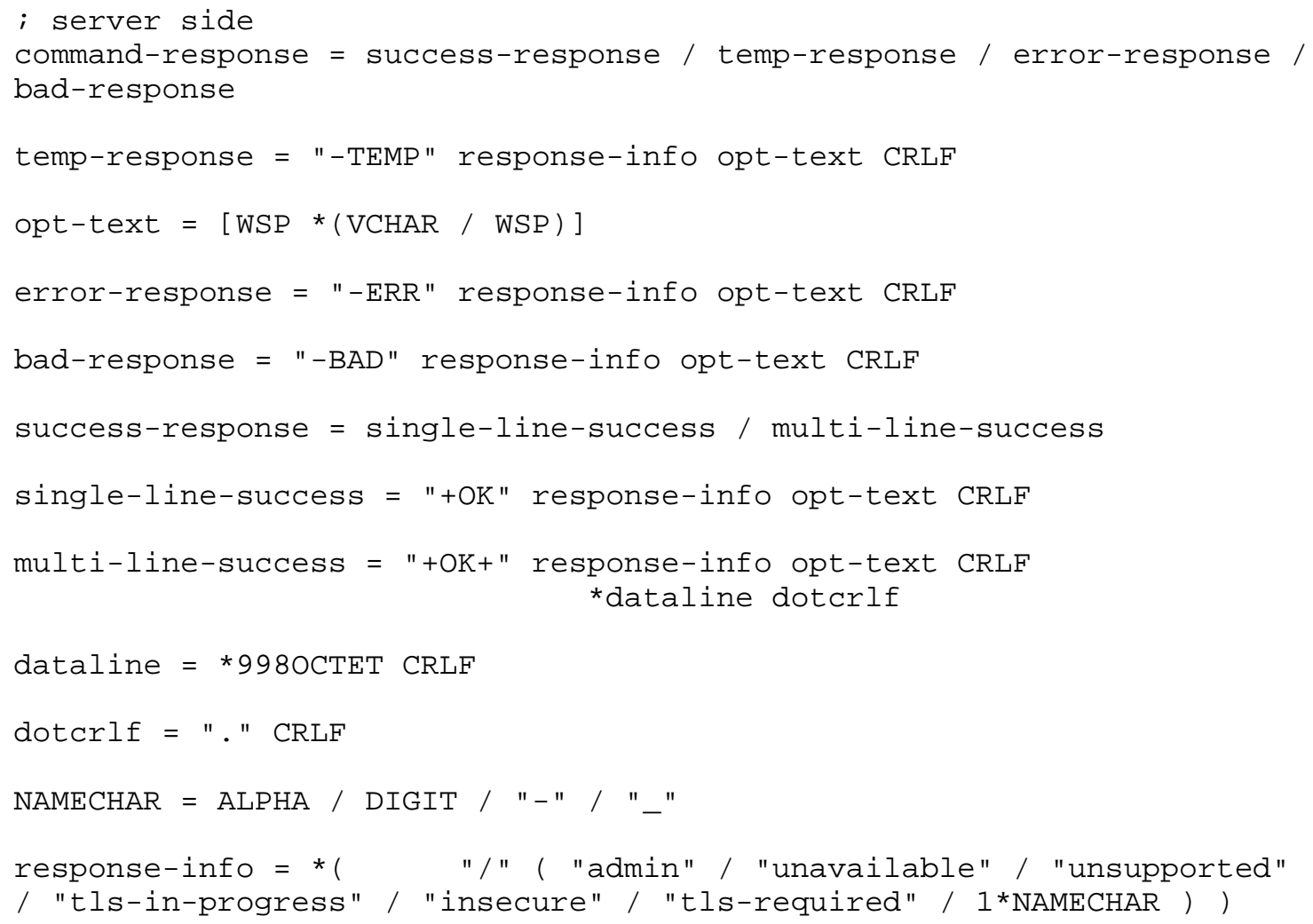

13. Acknowledgements

The description of STARTTLS is based on [RFC-SMTP-TLS]. 
14. References

14.1. Normative References

\begin{tabular}{|c|c|}
\hline [RFC-MIME ] & $\begin{array}{l}\text { Freed, N. and N. Borenstein, "Multipurpose } \\
\text { Internet Mail Extensions (MIME) Part One: Format } \\
\text { of Internet Message Bodies", RFC 2045, November } \\
1996 .\end{array}$ \\
\hline$[\mathrm{RFC}-\mathrm{ABNF}]$ & $\begin{array}{l}\text { Crocker, D., Ed. and P. Overell, "Augmented BNF } \\
\text { for Syntax Specifications: ABNF", RFC 2234, } \\
\text { November } 1997 .\end{array}$ \\
\hline$[\mathrm{RFC}-\mathrm{SRV}]$ & $\begin{array}{l}\text { Gulbrandsen, A., Vixie, P., and L. Esibov, "A DNS } \\
\text { RR for specifying the location of services (DNS } \\
\text { SRV)", RFC 2782, February } 2000 .\end{array}$ \\
\hline [RFC-SMTP ] & $\begin{array}{l}\text { Klensin, J., "Simple Mail Transfer Protocol", RFC } \\
2821 \text {, April } 2001 .\end{array}$ \\
\hline [RFC-SMTPEXT] & $\begin{array}{l}\text { Myers, J., "SMTP Service Extension for } \\
\text { Authentication", RFC 2554, March } 1999 .\end{array}$ \\
\hline [RFC-MTRK-ESMTP ] & $\begin{array}{l}\text { Allman, E. and T. Hansen, "SMTP Service Extension } \\
\text { for Message Tracking", RFC 3885, September } 2004 \text {. }\end{array}$ \\
\hline [RFC-MTRK-MODEL ] & $\begin{array}{l}\text { Hansen, T., "Message Tracking Models and } \\
\text { Requirements", RFC 3885, September } 2004 \text {. }\end{array}$ \\
\hline$[\mathrm{RFC}-\mathrm{MTRK}-\mathrm{TSN}]$ & $\begin{array}{l}\text { Allman, E., "The Message/Tracking-Status MIME } \\
\text { Extension", RFC 3886, September } 2004 .\end{array}$ \\
\hline [RFC-URI ] & $\begin{array}{l}\text { Berners-Lee, T., Fielding, R. and L. Masinter, } \\
\text { "Uniform Resource Identifiers (URI): Generic } \\
\text { Syntax", RFC 2396, August 1998. }\end{array}$ \\
\hline$[\mathrm{LS}]$ & $\begin{array}{l}\text { Dierks, T. and C. Allen, "The TLS Protocol Version } \\
\text { 1.0", RFC } 2246 \text {, January } 1999 .\end{array}$ \\
\hline
\end{tabular}




\subsection{Informational References}

[BCP 35 ]

[RFC-SHA1 ]

[RFC-KEYWORDS ]

$[\mathrm{RFC}-\mathrm{SMTP}-\mathrm{TLS}]$

$[\mathrm{RFC}-\mathrm{X} 509]$

[POP 3 ]

[NNTP ]
Petke, R. and I. King, "Registration Procedures for URL Scheme Names", BCP 35, RFC 2717, November 1999.

Eastlake, D. and P. Jones, "US Secure Hash Algorithm 1 (SHA1)", RFC 3174, September 2001.

Bradner, S., "Key words for use in RFCs to Indicate Requirement Levels", BCP 14, RFC 2119, March 1997.

Hoffman, P., "SMTP Service Extension for Secure SMTP over Transport Layer Security", RFC 3207 , February 2002 .

Housley, R., Polk, W., Ford, W. and D. Solo, "Internet X.509 Public Key Infrastructure Certificate and Certificate Revocation List (CRL) Profile", RFC 3280, April 2002.

Myers, J. and M. Rose, "Post Office Protocol Version 3", STD 53, RFC 1939, May 1996.

Kantor, B. and P. Lapsley, "Network News Transfer Protocol", RFC 977, February 1986. 


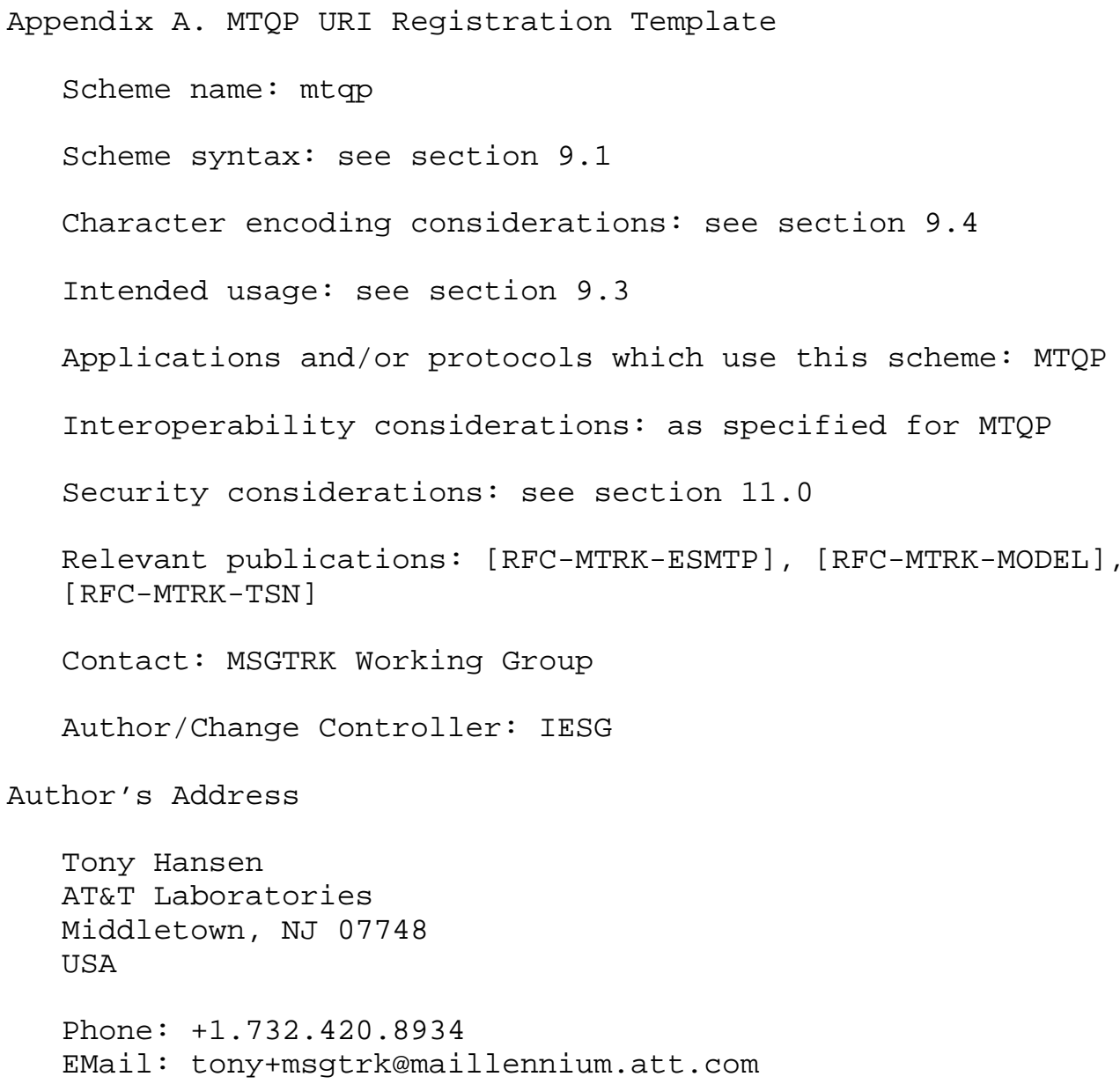


Full Copyright statement

Copyright (C) The Internet Society (2004).

This document is subject to the rights, licenses and restrictions contained in BCP 78, and except as set forth therein, the authors retain all their rights.

This document and the information contained herein are provided on an "AS IS" basis and THE CONTRIBUTOR, THE ORGANIZATION HE/S HE REPRESENTS OR IS SPONSORED BY (IF ANY), THE INTERNET SOCIETY AND THE INTERNET ENGINEERING TASK FORCE DISCLAIM ALL WARRANTIES, EXPRESS OR IMPLIED, INCLUDING BUT NOT LIMITED TO ANY WARRANTY THAT THE USE OF THE INFORMATION HEREIN WILL NOT INERINGE ANY RIGHTS OR ANY IMPLIED WARRANTIES OF MERCHANTABILITY OR FITNESS FOR A PARTICULAR PURPOSE.

Intellectual Property

The IETF takes no position regarding the validity or scope of any Intellectual property Rights or other rights that might be claimed to pertain to the implementation or use of the technology described in this document or the extent to which any license under such rights might or might not be available; nor does it represent that it has made any independent effort to identify any such rights. Information on the IETF's procedures with respect to rights in IETF Documents can be found in $\mathrm{BCP} 78$ and $\mathrm{BCP} 79$.

Copies of IPR disclosures made to the IETF Secretariat and any assurances of licenses to be made available, or the result of an attempt made to obtain a general license or permission for the use of such proprietary rights by implementers or users of this specification can be obtained from the IETF on-line IPR repository at http://www.ietf.org/ipr.

The IETF invites any interested party to bring to its attention any copyrights, patents or patent applications, or other proprietary rights that may cover technology that may be required to implement this standard. Please address the information to the IETF at ietfipreietf.org.

Acknowledgement

Funding for the RFC Editor function is currently provided by the Internet Society. 